\title{
BMJ Open Risk factors associated with premature myocardial infarction: a systematic review protocol
}

\author{
Sagar B Dugani, ${ }^{1,2}$ Ana Patricia Ayala Melendez, ${ }^{3}$ Roger Reka, ${ }^{3}$ Yousif M Hydoub, ${ }^{4}$ \\ Shannon N McCafferty, ${ }^{5}$ M Hassan Murad, ${ }^{6}$ Alawi A Alsheikh-Ali, ${ }^{7,8}$ \\ Samia Mora ${ }^{2,9,10}$
}

To cite: Dugani SB, Ayala Melendez AP, Reka R, et al. Risk factors associated with premature myocardial infarction: a systematic review protocol. BMJ Open 2019:9:e023647. doi:10.1136/ bmjopen-2018-023647

- Prepublication history and additional material for this paper are available online. To view these files, please visit the journal online (http://dx.doi. org/10.1136/bmjopen-2018023647).

AAA and SM are co-senior investigators.

Received 16 April 2018 Revised 9 December 2018 Accepted 13 December 2018

Check for updates

(c) Author(s) (or their employer(s)) 2019. Re-use permitted under CC BY-NC. No commercial re-use. See rights and permissions. Published by BMJ.

For numbered affiliations see end of article.

Correspondence to Dr Sagar B Dugani; dugani.chandrasagar@mayo. edu

\section{ABSTRACT}

Introduction Premature myocardial infarction (MI) generally refers to $\mathrm{Ml}$ in men $\leq 55$ years or women $\leq 65$ years. Premature $\mathrm{Ml}$ is a major contributor to cardiovascular disease (CVD), which claimed 17.6 million lives globally in 2016. Reducing premature MI and CVD is a key priority for all nations; however, there is sparse synthesis of information on risk factors associated with premature MI. To address this knowledge gap, we are conducting a systematic review to describe the association between risk factors (demographics, lifestyle factors and biomarkers) and premature $\mathrm{Ml}$.

Methods and analysis The following databases were searched from inception to June 2018: CENTRAL, CINAHL, Clinical Trials, EMBASE and MEDLINE. We will include original research articles (case-control, cohort and crosssectional studies) that report a quantitative relationship between at least one risk factor and premature MI. Two investigators will use predetermined selection criteria and independently screen articles based on title and abstract (primary screening). Articles that meet selection criteria will undergo full-text screening based on criteria used for primary screening (secondary screening). Data will be extracted using predetermined data extraction forms. The Newcastle-0ttawa Scale for case-control and cohort studies will be used to evaluate the risk of bias and will be adapted for cross-sectional studies. Whenever feasible, data will be summarised into a random-effects metaanalysis.

Ethics and dissemination To our knowledge, this will be the first study to synthesise results on the relationship between risk factors and premature Ml. These findings will inform healthcare providers on factors associated with risk of premature $\mathrm{Ml}$ and potentially improve primary prevention efforts by guiding development of interventions. These findings will be summarised and presented at conferences and through publication in a peer-reviewed journal.

PROSPERO registration number CRD42018076862.

\section{INTRODUCTION}

Non-communicable diseases (NCDs), which generally include cardiovascular disease (CVD), diabetes, respiratory disease and cancer, are a major driver of global mortality. ${ }^{1}$ The WHO estimated that NCDs annually

\section{Strengths and limitations of this study}

- This will be the first comprehensive, systematic review of diverse risk factors associated with premature myocardial infarction (MI).

- The broad search strategy will identify infrequently reported risk factors and identify new strategies to predict risk of premature $\mathrm{Ml}$.

- This study will categorise results by world region, thereby identifying regional variation in association of risk factors with premature MI.

- This study will only identify English-language articles from the peer-reviewed literature.

- This study will exclude articles with fewer than 100 cases of premature MI.

claim approximately 15 million lives within the age group of 30-70 years (termed 'premature mortality'), with the major burden concentrated in low-income and middle-income countries. $^{2}$

CVD is the major contributor to NCD-related morbidity and mortality, and in 2016, it claimed 17.6 million lives globally. ${ }^{3}$ The majority of CVD-related deaths comes from ischaemic heart disease, which is composed of acute myocardial infarction (MI) and ischaemic heart failure. ${ }^{4}$ In 2016, ischaemic heart disease (IHD) claimed 9.5 million lives, a 19\% increase over the prior decade, attributed to ageing and a growing population. ${ }^{3}$ The high burden of IHD, particularly among young adults, adversely affects personal and economic productivity, health of caregivers and increases healthcare costs. ${ }^{156}$ Reducing the burden of IHD is a key priority for all countries.

Although premature mortality refers to mortality in the age group of 30-70 years, premature MI generally refers to MI in men $\leq 55$ years or women $\leq 65$ years. $^{7-9}$ Although diagnosis, management and treatment of MI have improved, results on IHD 
mortality in young adults have been mixed. A US study showed that IHD mortality in young women ( $<55$ years) has remained unchanged over a 20-year period from 1990 to $2011 .{ }^{10}$ Similarly, in countries including Australia, Canada and Scotland, young adults have shown minimal to no improvement, higher IHD mortality, or different IHD mortality for men versus women. ${ }^{11-16}$ The reasons for suboptimal IHD mortality in young adults are incompletely understood and require further characterisation.

There are limited international studies on the relationship between risk factors (demographics, lifestyle factors, clinical risk factors and biomarkers) and premature MI. The INTERHEART study used a case-control design on 27098 adults from 52 countries to assess the relationship between risk factors and acute MI. Although not designed to exclusively study premature MI, this study showed that the population attributable risk of nine risk factors (lifestyle factors (smoking; consumption of fruit and vegetables; exercise; consumption of alcohol; psychosocial stress); clinical risk factors (hypertension; diabetes; abdominal obesity) and, biomarkers (ratio of blood levels of apolipoprotein B/apolipoprotein A1 (apoB/apoA)) was higher among younger ( $\leq 55$ years for men and $\leq 65$ years for women) versus older adults. ${ }^{17}$ The Global Registry of Acute Coronary Events (GRACE) study evaluated 24165 individuals from 14 countries presenting with acute coronary syndrome (ACS; comprised of MI and unstable angina) and assessed prevalence of risk factors in 10-year age groups ( $<45$ years up to $\geq 85$ years). They showed variable age-related trends for different risk factors (demographics (higher proportion of men in younger age groups); lifestyle factors (higher prevalence of smoking in younger individuals); clinical risk factors (lower prevalence of hypertension in younger individuals; prevalence of diabetes peaked in the 65-74 years age group); biomarkers (prevalence of hyperlipidaemia peaked in the 55-64years age group).$^{18}$

In addition to INTERHEART and GRACE, other studies have evaluated the relationship between select risk factors and ACS. With respect to lifestyle factors, studies based in Spain and the Middle East have shown higher prevalence of smoking in younger individuals with ACS. ${ }^{19-22}$ Smoking was generally more prevalent in men versus women, although this was not observed in a large study based in Canada. ${ }^{23}$ In addition to lifestyle factors, few studies have examined the relationship between biomarkers and premature MI. Higher levels of low-density lipoprotein (LDL) cholesterol and triglycerides, and lower levels of high-density lipoprotein (HDL) cholesterol were observed in younger versus older individuals with ACS in the Middle East ${ }^{20}$; levels of high-sensitivity C reactive protein (hsCRP), a marker of inflammation, were persistently higher in younger women versus younger men $^{24}$ and levels of ferritin were higher among individuals with premature MI versus controls. ${ }^{25}$

To our knowledge, there are no published systematic reviews that describe the association between risk factors and premature MI. To address this gap, we have developed a protocol for a systematic review to describe the relationship between risk factors (demographics, lifestyle factors and biomarkers) and premature MI. From these articles, we also describe the relationship between clinical risk factors and premature MI.

\section{METHODS AND ANALYSIS \\ Standards}

We will adhere to the Preferred Reporting Items for Systematic Reviews and Meta-Analyses (PRISMA) Statement for the completion of the systematic review. We have adhered to PRISMA protocols (PRISMA-P) for the reporting of this protocol (online supplementary appendix A). ${ }^{26}$

\section{Protocol and registration}

This review protocol is registered online with the International Prospective Register of Systematic Reviews. The online profile will be revised for major protocol amendments.

We searched OVID MEDLINE (1946 to June 2018, including epub ahead of print, in process and other non-indexed citations), OVID EMBASE (1947 to June 2018), EBSCO CINAHL Plus with full text (1981 to June 2018), OVID AMED (1985 to June 2018), ClinicalTrials.gov and Cochrane Central to identify articles on premature MI. The search strategy was developed by an academic health sciences librarian (APAM) with input from the study investigators. The search strategy was translated using each database platform's command language, controlled vocabulary and search fields. MeSH terms, EMTREE terms, CINAHL headings and text words were used for the search concepts of MI, young adults and middle-aged adults. The concept of premature was captured using text words. MeSH headings included: MI, ACS, young adult, adult and middle aged.

A multi-stranded approach was used to search the concept of MI in MEDLINE, EMBASE, CINAHL and Cochrane Central: search terms for MI, heart attack, ST segment elevation MI (STEMI), non-ST segment elevation MI (NSTEMI) and ACS were combined with Boolean OR (strand 1). Search terms for young adults, adults, middle aged were combined with the Boolean OR (strand 2). Search terms for the concept of premature, untimeliness and early onset were combined with the Boolean OR (string 3). Finally, all strings were combined with the Boolean AND. Our search strategy intentionally did not include risk factors to allow for identification of infrequently described risk factors. All searches were limited to the English language.

Searches were completed by June 2018. The MEDLINE search strategy is shown in online supplementary appendix B.

\section{Eligibility criteria}

Participants

Men (18-55 years) and women (18-65 years) who have experienced an MI. 


\section{Risk factors}

Demographics, lifestyle factors, clinical risk factors and biomarkers. Demographics include sex, race or ethnicity, education, income, living area (urban vs rural) and family history of cardiac disease. Lifestyle factors include exercise or physical activity, diet, alcohol consumption, tobacco use including shisha and khat, recreational drug use and psychological stress. Clinical risk factors include diabetes, hypertension, obesity and dyslipidaemia. Biomarkers include, but are not restricted to, serum cholesterol (total cholesterol, HDL cholesterol, non-HDL cholesterol, LDL cholesterol), triglycerides, lipoprotein (a), apolipoprotein (apo) A, apoB, liver enzymes (aspartate transaminase, alanine transaminase), inflammatory markers (erythrocyte sedimentation rate, $\mathrm{C}$ reactive protein, hsCRP), fasting glucose, haemoglobin Alc (HbAlc) and other markers, including kallikrein, matrix metallopeptidase 9 (MMP9), galectin-3, protein $\mathrm{S}$, protein $\mathrm{C}$, citrate, valine, leucine, isoleucine, alanine, homocysteine, fibrinogen and iron.

\section{Myocardial infarction}

The following events will be included: ACS, STEMI, non-ST elevation-ACS, or NSTEMI. The following events will be excluded: stable coronary heart disease, stable atherosclerosis, stable coronary artery disease and heart failure.

\section{Study design}

Research articles published in English and that report a quantitative relationship between at least one risk factor (demographics, lifestyle factors, or biomarkers) and premature MI. From these studies, we will also assess the relationship between clinical risk factors and premature MI. Articles that exclusively evaluate clinical risk factors but no other risk factors (demographics, lifestyle factors, or biomarkers) will be described qualitatively but will not be included in quantitative analyses. We will include casecontrol, cohort and cross-sectional studies. The following article types will be excluded: conference abstracts, review papers, systematic reviews, reports from organisations, research thesis (eg, post-graduate, graduate, or undergraduate theses or reports), qualitative articles (eg, focus groups, interviews, or articles that do not provide a quantitative relationship between risk factors and premature $\mathrm{MI}$ ), editorials, commentaries, opinion pieces, letters to the editor, viewpoints and case reports. We will exclude articles with fewer than 100 cases of premature MI.

\section{Creation of database}

We used search terms (developed in consultation with an academic health sciences librarian) to identify articles from electronic research databases. Duplicate results were removed and articles from different databases were merged into a single database and imported to Covidence, an online software product for systematic reviews based on the Cochrane Review process.

\section{Screening, data extraction and risk of bias}

For primary screening, the title and abstract were used to identify eligible studies based on selection criteria described above. For secondary screening, full-text articles were screened using selection criteria for primary screening. If an article meets selection criteria, but is not available online, we will make two attempts to contact authors by electronic mail and obtain the full text. Despite this, if the full-text is not available, we will exclude the study. Only English-language articles will be included, which may influence the precision of pooled estimates but not necessarily result in systematic bias. ${ }^{27}$ Dual abstract and full-text screening will be done using standardised forms.

After a final set of articles is obtained, information will be extracted independently by two investigators using a standardised data extraction form. The form will include the following information: study type, study sites (countries), year of study, year of publication, number of participants, risk factor examined and relationship of risk factor with premature MI (eg, prevalence, incidence rate, relative risk, odds ratio). We will include risk factors prior to and up to 4 weeks following premature MI, as risk factors, particularly biomarkers, following that period may not reflect the acute MI phase and also may be modified by pharmacological treatment. Where possible, we will analyse studies by sex and segregate by world regions defined by the World Bank. ${ }^{28}$

For the final set of articles, the Newcastle-Ottawa Scale for case-control and cohort studies will be used to evaluate the risk of bias, and will be adapted for cross-sectional studies. ${ }^{29}$ Studies that do not adjust risk factors for potentially confounding variables will be considered to have higher risk of bias.

For primary and secondary screening, data extraction and risk of bias assessment, conflicts between reviewers will be resolved by discussion between reviewers, and through consultation with a third reviewer, if necessary.

\section{Data synthesis}

Based on the quality of the studies that meet final study criteria, we will determine feasibility to conduct a meta-analysis to determine the relationship between risk factors and premature MI. Where applicable, we will present sex-stratified analyses.

If the relative association measures have similar or close adjustment, we will conduct a random-effects meta-analysis (as implemented in the DerSimonian and Laird method) to pool across studies. ${ }^{30}$ The random-effects model is chosen a priori due to expected variation of studies populations and settings. We will use the $\mathrm{I}^{2}$ index and Cochrane $Q$ test to determine the extent of heterogeneity and whether it is attributable to chance. ${ }^{31}$ We will use STATA V.15 or higher to conduct analyses. Subgroup analyses based on sex or other relevant factors will be conducted and tested using an interaction test as described. ${ }^{32}$ 
Although publication bias assessment methods have been developed for randomised controlled trials, we will attempt to evaluate publication bias in cases where we have more than 10 studies in a meta-analysis. We will visually inspect the funnel plots for symmetry and conduct the Egger regression test. ${ }^{33}$

\section{Summarising evidence}

Based on the Grading of Recommendations, Assessment, Development and Evaluations (GRADE) Working Group recommendations, we will use GRADEpro (https://community.cochrane.org/help/tools-andsoftware/gradepro-gdt) to build evidence of profiles that meet final eligibility criteria. We will evaluate the quality of evidence (certainty in estimates) using the GRADE approach for each association separately. We will examine the limitations of inclusion studies and whether any elements of indirectness, inconsistency, imprecision or publication bias were present. We will consider increasing the certainty level if the association is strong (ie, large relative effect size over 2.0).

We will include the following elements: number of participants and country where the study is based; type of study (eg, case-control, prospective cohort); prevalence and association of risk factors with premature MI, including relationships that are not statistically significant; analysis of prevalence and association of risk factors by sex and by world region and additional comments or notes related to the study. If data are available, we will conduct a subgroup analysis based on different age thresholds or other factors.

\section{Current study status}

At the time of writing this manuscript, primary and secondary screening was completed independently by two investigators and data extraction was in progress.

\section{Ethics and dissemination}

This study involves analysis of data from published literature and does not involve individual-level identifiable data. Given this, there were no privacy concerns that required ethical approval.

Results from this study will be presented at conferences and through publication in a peer-reviewed journal.

\section{Patient and public involvement}

There was no patient or public involvement in this study.

\section{DISCUSSION}

To our knowledge, this will be the first systematic review on the relationship between risk factors and premature MI. Information from this study may be useful for policy makers, government and non-government stakeholders and healthcare providers to develop and implement strategies to reduce the burden of risk factors, and consequently, of premature MI, IHD and CVD.
Several global declarations have focused on tackling the growing NCD burden. For instance, the United Nations World Health Assembly adopted the '25 ×25' resolution to reduce premature NCD-related mortality by $25 \%$ by the year $2025^{2}$ and the United Nations sustainable development goals aim to reduce premature NCD-related mortality by one-third by the year $2030 .{ }^{3435}$ In this context, our study will identify knowledge gaps with sparse information on risk factors. This will guide development of a strategic research programme to address the rising burden of premature MI and CVD. This is particularly relevant given the sobering update that many countries are not on track to achieve their NCD targets. ${ }^{2} 36$

\section{Author affiliations}

${ }^{1}$ Division of Hospital Internal Medicine, Mayo Clinic, Rochester, Minnesota, USA ${ }^{2}$ Center for Lipid Metabolomics, Brigham and Women's Hospital, and Harvard Medical School, Boston, Massachusetts, USA

${ }^{3}$ Gerstein Science Information Centre, University of Toronto, Toronto, Ontario, Canada

${ }^{4}$ Al-Mafraq Hospital, Abu Dhabi, United Arab Emirates

${ }^{5}$ University of Pittsburgh, Pittsburgh, Pennsylvania, USA

${ }^{6}$ Evidence-Based Practice Center, Mayo Clinic, Rochester, Minnesota, USA

${ }^{7}$ College of Medicine, Mohammed Bin Rashid University of Medicine and Health Sciences, Dubai, United Arab Emirates

${ }^{8}$ Sheikh Khalifa Medical City, Abu Dhabi, United Arab Emirates

${ }^{9}$ Division of Cardiovascular Medicine, Brigham and Women's Hospital, and Harvard Medical School, Boston, Massachusetts, USA

${ }^{10}$ Division of Preventive Medicine, Brigham and Women's Hospital, and Harvard Medical School, Boston, Massachusetts, USA

Acknowledgements We thank Dr Edward Callachan and Dr Arshia Shaikh (both from Sheikh Khalifa Medical City, Abu Dhabi, United Arab Emirates) for initial assistance with the systematic review.

Contributors Conceptualisation: AAA, SBD and SM. Formal analysis: APAM, RR, SBD, SNM and YMH. Investigation: APAM and RR. Methodology: AAA, APAM, MHM, SBD, YMH and SM. Project administration: SBD. Supervision: AAA and SM. Validation: SBD and YMH. Writing-original draft: APAM, RR and SBD. Writing-review and editing: AAA, APAM, MHM, RR, SBD, SM, SNM and YMH. Guarantor: AAA, SBD and SM. All authors contributed to manuscript revisions and approved the draft submitted.

Funding This study was funded by NHLBI grant K24 HL136852 to SM and by a United Arab Emirates-Harvard Medical School Cooperative Research Award (Dubai, United Arab Emirates) to SM and AAA. The funders' and authors' institutions were not involved in developing this protocol or in the decision to submit this manuscript for publication.

Competing interests None declared.

Patient consent for publication Not required.

Provenance and peer review Not commissioned; externally peer reviewed.

Open access This is an open access article distributed in accordance with the Creative Commons Attribution Non Commercial (CC BY-NC 4.0) license, which permits others to distribute, remix, adapt, build upon this work non-commercially, and license their derivative works on different terms, provided the original work is properly cited, appropriate credit is given, any changes made indicated, and the use is non-commercial. See: http://creativecommons.org/licenses/by-nc/4.0/.

\section{REFERENCES}

1. Hunter DJ, Reddy KS. Noncommunicable diseases. N Engl J Med 2013;369:1336-43.

2. WHO. Noncommunicable Diseases Progress Monitor 2017. 2017.

3. Abajobir AA, Abbafati C, Abbas KM, et al. Global, regional, and national age-sex specific mortality for 264 causes of death, 1980- 
2016: a systematic analysis for the Global Burden of Disease Study 2016. Lancet 2017;390:1151-210.

4. Moran AE, Forouzanfar MH, Roth GA, et al. The global burden of ischemic heart disease in 1990 and 2010: the Global Burden of Disease 2010 study. Circulation 2014;129:1493-501.

5. OECD. OECD Health Policy Studies: cardiovascular disease and diabetes. 2015 http://www.oecd.org/els/health-systems/hcqicardiovascular-disease-and-diabetes.htm

6. AHA. Cardiovascular disease: a costly burden for America. 2017 https://www.heart.org/idc/groups/heart-public/@wcm/@adv/ documents/downloadable/ucm_491543.pdf

7. De Sutter J, De Bacquer D, Kotseva K, et al. Screening of family members of patients with premature coronary heart disease; results from the EUROASPIRE II family survey. Eur Heart J 2003;24:249-57.

8. Kotseva K, Wood D, De Backer G, et al. EUROASPIRE III: a survey on the lifestyle, risk factors and use of cardioprotective drug therapies in coronary patients from 22 European countries. Eur $\mathrm{J}$ Cardiovasc Prev Rehabil 2009;16:121-37.

9. Lloyd-Jones DM, Nam BH, D'Agostino RB, et al. Parental cardiovascular disease as a risk factor for cardiovascular disease in middle-aged adults: a prospective study of parents and offspring. JAMA 2004;291:2204-11.

10. Wilmot KA, O'Flaherty M, Capewell S, et al. Coronary heart disease mortality declines in the United States From 1979 Through 2011 clinical perspective. Circulation 2015;132:997-1002.

11. Allender S, Scarborough $\mathrm{P}, \mathrm{O}$ 'Flaherty $\mathrm{M}$, et al. Patterns of coronary heart disease mortality over the 20th century in England and Wales: Possible plateaus in the rate of decline. BMC Public Health 2008;8:148.

12. Ford ES, Capewell S. Coronary heart disease mortality among young adults in the U.S. from 1980 through 2002: concealed leveling of mortality rates. J Am Coll Cardiol 2007;50:2128-32.

13. Gupta A, Wang Y, Spertus JA, et al. Trends in acute myocardial infarction in young patients and differences by sex and race, 2001 to 2010. J Am Coll Cardiol 2014;64:337-45.

14. Izadnegahdar M, Singer J, Lee MK, et al. Do younger women fare worse? Sex differences in acute myocardial infarction hospitalization and early mortality rates over ten years. $J$ Womens Health 2014;23:10-17.

15. Nedkoff LJ, Briffa TG, Preen DB, et al. Age- and sex-specific trends in the incidence of hospitalized acute coronary syndromes in Western Australia. Circ Cardiovasc Qual Outcomes 2011;4:557-64.

16. O'Flaherty M, Ford E, Allender S, et al. Coronary heart disease trends in England and Wales from 1984 to 2004: concealed levelling of mortality rates among young adults. Heart 2008;94:178-81.

17. Yusuf S, Hawken S, Ounpuu S, et al. Effect of potentially modifiable risk factors associated with myocardial infarction in 52 countries (the INTERHEART study): case-control study. Lancet 2004;364:937-52.

18. Avezum A, Makdisse M, Spencer F, et al. Impact of age on management and outcome of acute coronary syndrome: observations from the Global Registry of Acute Coronary Events (GRACE). Am Heart J 2005;149:67-73.

19. Panduranga P, Sulaiman K, Al-Zakwani I, et al. Acute coronary syndrome in young adults from oman: results from the gulf registry of acute coronary events. Heart Views 2010;11:93.
20. Ahmed E, Alhabib KF, El-Menyar A, et al. Age and clinical outcomes in patients presenting with acute coronary syndromes. $J$ Cardiovasc Dis Res 2013;4:134-9.

21. Morillas P, Bertomeu V, Pabón P, et al. Characteristics and outcome of acute myocardial infarction in young patients. The PRIAMHO II study. Cardiology 2007;107:217-25.

22. El-Menyar A, Zubaid M, Shehab A, et al. Prevalence and impact of cardiovascular risk factors among patients presenting with acute coronary syndrome in the middle East. Clin Cardiol 2011;34:51-8.

23. Pelletier R, Humphries KH, Shimony A, et al. Sex-related differences in access to care among patients with premature acute coronary syndrome. CMAJ 2014;186:497-504.

24. Lu Y, Zhou S, Dreyer RP, et al. Sex differences in lipid profiles and treatment utilization among young adults with acute myocardial infarction: Results from the VIRGO study. Am Heart $J$ 2017;183:74-84.

25. Iqbal MP, Mehboobali N, Tareen AK, et al. Association of body iron status with the risk of premature acute myocardial infarction in a Pakistani population. PLoS One 2013;8:e67981.

26. Shamseer L, Moher D, Clarke M, et al. Preferred reporting items for systematic review and meta-analysis protocols (PRISMA-P) 2015: elaboration and explanation. BMJ 2015;349:g7647.

27. Morrison A, Polisena J, Husereau D, et al. The effect of Englishlanguage restriction on systematic review-based meta-analyses: a systematic review of empirical studies. Int $J$ Technol Assess Health Care 2012;28:138-44

28. World Bank Group. World Bank country and lending groups. 2018 https://data.worldbank.org/region/middle-east-and-north-africa? view=chart (Accessed 26 Apr 2018).

29. The Newcastle-Ottawa Scale. The Newcastle-Ottawa Scale (NOS) for assessing the quality of nonrandomised studies in meta-analyses. 2018 http://www.ohri.ca/programs/clinical_epidemiology/oxford.asp (Accessed 12 Sep 2018).

30. DerSimonian R, Laird N. Meta-analysis in clinical trials. Control Clin Trials 1986;7:177-88.

31. Higgins JP, Thompson SG, Deeks JJ, et al. Measuring inconsistency in meta-analyses. BMJ 2003;327:557-60.

32. Altman DG, Bland JM. Interaction revisited: the difference between two estimates. BMJ 2003;326:219.

33. Egger M, Davey Smith G, Schneider M, et al. Bias in meta-analysis detected by a simple, graphical test. BMJ 1997;315:629-34

34. WHO. World Health Organization: Global strategy for the prevention and control of noncommunicable diseases. 2000 http://apps.who.int/ gb/archive/pdf_files/WHA53/ea14.pdf

35. SDG. Sustainable development knowledge platform. https://sustaina bledevelopment.un.org/post2015/transformingourworld

36. Bennett JE, Stevens GA, Mathers CD, et al. NCD Countdown 2030: worldwide trends in non-communicable disease mortality and progress towards Sustainable Development Goal target 3.4. Lancet 2018;392:1072-88. 